\title{
Intelligence on Optical Transport SDN
}

\author{
Xu Chen and Yongiian Zhang
}

\begin{abstract}
Telecom technologies have been developed very fast in these years. To meet the new trends and requirements of telecom applications, the optical transport network needs to be restructured with more dynamic, more cost-efficient, softer and more intelligent technologies. SDN accompanied with OpenFlow protocol is a good choice. In this paper, we study the optical transport network with intelligence view, and detailed the intelligence requirement gaps between current optical transport networks and new trends of telecom applications firstly, then detailed what major intelligent technologies can be used to build such an optical transport networks and survey current innovative researches for solving such gaps, and finally discuss the potential intelligence technologies which will be used to optimize the SDN optical networks in the next step.
\end{abstract}

Index Terms - Intelligent, OpenFlow, optical transport, SDN, algorithms.

\section{INTRODUCTION}

Global telecom industry witnesses the unlimited bandwidth growth and increased preference of new technologies; hot words like Big Data, Cloud, Advanced LTE and NG-PON2 are bound up with our life. These new technologies and applications are featured with dynamic, insatiable bandwidth demand, unpredictable traffic patterns, and rising peak-to-average ratios. A more intelligent optical transport network with more data-rate flexible, agile, reconfigurable, and resource-efficient is required to guarantee the service quality and performance for such trends.

Optical transport networks have been developed from SONET/SDH to MSTP, DWDM and ASON/GMPLS. Many vendors already can provide systems with $100 \mathrm{~Gb} / \mathrm{s}$ bitrates, and there are experiments in $400 \mathrm{G}$ and $1 \mathrm{~Tb} / \mathrm{s}$ per wavelength. Optical transport networks had involved many intelligence technologies on the functions of transport, multiplexing, routing, management, supervision and survivability. But there are gaps to meet the aforementioned new trends of telecom applications.

SDN (Soft Defined Network) is another hot technology and has solved the above problems for switch networks, many researchers also have been dedicated to involving SDN technology on optical transport network, and had got good achievements.

In this paper, we study the optical transport SDN technology with intelligence view, and from our best knowledge, it is the first work using that view. Section II detailed the intelligence requirement gaps between current

Manuscript received May 15, 2014; revised August 26, 2014.

$\mathrm{Xu}$ Chen is with the Premier Farnell, Chengdu 610073, China (e-mail: cdcxchenxu@gmail.com).

Yong Jian Zhang is with University of International Relation, Beijing 10086, China (e-mail: zyjbaba@163.com). optical transport networks and new trends of telecom applications, Section III detailed the major intelligent technologies of optical networks for solving such gaps, Section IV survey the innovative researches for building an optical transport SDN's architecture, unified control plane platform and detailed algorithms or mechanisms, and Section $\mathrm{V}$ discussed the potential intelligence technologies which will be used to optimize the SDN optical networks in the next step.

\section{INTELLIGENT REQUIREMENT GAPS OF CURRENT OPTICAL NETWORKS}

New telecom application challenges for current optical networks can be summarized as efficiency and priceperformance, the ultimately goal and drivers are to improve these two points. Major gaps between the new application requirements and current optical networks will focus on optical layer and control plane.

For many years, optical transport network are providing rigid bandwidth when traffic was created, but setting up or updating new application need reconfigurable and flexible bandwidth. Now setting a new service need very complicate processes with weeks or even month's period, and the transport network price-performance is decreasing more slowly than the other electronic routing and switching.

Network resource provisioning across multi-layers is not optimized when each layer is optimized in isolation. Events such as failures and network upgrades cannot be detected and mapped across independent control layers causing higher network maintenance and diagnosis complexities. There are many challenges to the network control plane including mechanism for impairments, signal aware routing and spectrum allocation, optical equalization, cross-layer optimizations, spectrum defragmentation, and network services and functions virtualization. Network slicing for transport layer and paths are set up with constraints on bandwidth, delay and jitter. It is difficult to allow transport networks in different domains to be intelligent interconnected and managed using dynamically controlled and coordinated multilayer network capabilities to meet service needs, and application layer cannot dynamically request and/or control network resources at wavelength, circuit, or flow granularity to seamlessly reconfigure resources based on higher-layer requirements.

In order to address the above requirements and issues, it is time to change the optical network to be soft, programmable and intelligent-controlled.

\section{BUILD AN INTELLIGENT OPTICAL TRANSPORT NETWORK}

As we mentioned before, to meet the major gaps, it needs focus on how to change the optical layer to be soft, 
programmable and intelligent, how to restructure an intelligent unified control plane.

\section{A. Programmable, Intelligent-Controlled Optical Layer}

Nowadays, major advanced technologies to support programmable and intelligent optical layer include intelligent and configurable transceivers, Flexible Grid DWDM, Hybrid EDFA-Raman amplifiers and Multi-degree ROADM.

With the advanced DSP technology involving in optical transceivers, modulation schemes (QPSK, BPSK, 16-QAM, OFDM), forward error correction (FEC) coding overhead (7, 13, 20, 25 percent), symbol rate, and number of carriers became configurable, under software control to achieve the desired performance. This features let the optic layer to be flexible and dynamic data rate on the lowest ends.

Recommendation ITU-T G.694.1 illustrate the Flexible Grid DWDM allowed frequency slots have a nominal central frequency (in THz) defined by $193.1+\mathrm{n} \times 0.00625$ where $\mathrm{n}$ is a positive or negative integer including 0 , and 0.00625 is the nominal central frequency granularity in $\mathrm{THz}$, and a slot width defined by: $12.5 \times \mathrm{m}$ where $\mathrm{m}$ is a positive integer and 12.5 is the slot width granularity in $\mathrm{GHz}$. Any combination of frequency slots is allowed as long as no two frequency slots overlap. This technology results in variable spectral width and the spectral efficiency on a link can be increased.

Hybrid EDFA-Raman amplifiers is an intelligent plug-and-play amplification product, it combines the advantages of EDFA which is with advanced intelligent embedded span diagnostics and channel monitoring features to enable operators to achieve OPEX savings through deployment efficiencies and autonomous plug-and-play operation.

Multi-degree ROADM with Colorless, Directionless, and Contentionless (CDC) features can improve the flexibility, wavelength blocking performance of ROADM, it allows add/drop signal be selected and directed at any terminated lines, termination of same wavelength coming from/going to different lines.

\section{B. Intelligent and Unified Control Plane}

Compared with the SONET/SDH constrained-free control plane, the current GMPLS control plane comprises topology discovery, path computation and signaling and provisioning functions, it can create the end-to-end light-paths under dynamic conditions automatically. Standardized interfaces allow the control plane to interact with client layer equipment, peer networks and service management applications. The use of a path computation element (PCE) allows centralized computation of the optical path and facilitates integration with other path computation elements for multi-layer and multi-domain operation. It is also an intelligent control plane.

But this protocol is overcomplicated. Network operators are reluctant in deploying such technologies in commercial networks. Furthermore, GMPLS is defined just over existing IP/MPLS networks and may not be capable of incorporating everything in a homogeneous protocol suite. Thus, GMPLS may not offer unified control plane infrastructure for future optical networks.

SDN architecture is introduced in which a control plane is extracted from the data plane of a physical hardware, and this control plane resides in a centralize controller. A centralized controller communicates with the data plane of physical hardware through an open protocol OpenFlow.

OpenFlow protocol extracts a common set of functionalities for heterogeneous switching granularity across multiple layers. The control plane decisions taken by the controller are represented in terms of a set of actions, rules, and policies those are cached in a data plane, and thus, the same data plane can support heterogeneous protocols and switching granularity. On the other hand, the controller abstracts the common-map of data planes across multiple layers while hiding the implementation details, and thus, offers virtualization.

Therefore, SDN architecture enabled with OpenFlow control protocols can simultaneously manage, control, and operate multiple layers with heterogeneous switching granularity. Such architecture enables more flexibility in hardware selections, shorter time to implement new technologies and products, more efficient and reliable automatic unified control, and optimized utilization of network resources.

As comparing SDN/OpenFlow with GMPLS control plane principles, the major differences and gaps are focused on hardware abstraction and virtualization, and the openness, efficiency, programmability and intelligence degrees. SDN structure is the better choice for optical transport network if it can be involved in optical control plane well.

In order to enable SDN/OpenFlow based unified control and management be involved in optical transport network control plane well, it need to extend OpenFlow from L2-L4 to Layer 0 (photonic) and Layer 1 (SONET/SDH, OTN). A typical hierarchical control architecture proposed in [1] is shown as Fig. 1, which enables control to be explicitly partitioned across the physical network resources, and OpenFlow are embedded in to ease the migration process or coexistence between legacy transport networks and OpenFlow based transport networks. A mediation application to translate to traditional transport management interfaces (e.g., TL1 or CORBA) can be introduced to allow legacy transport domains to operate in an SDN environment.

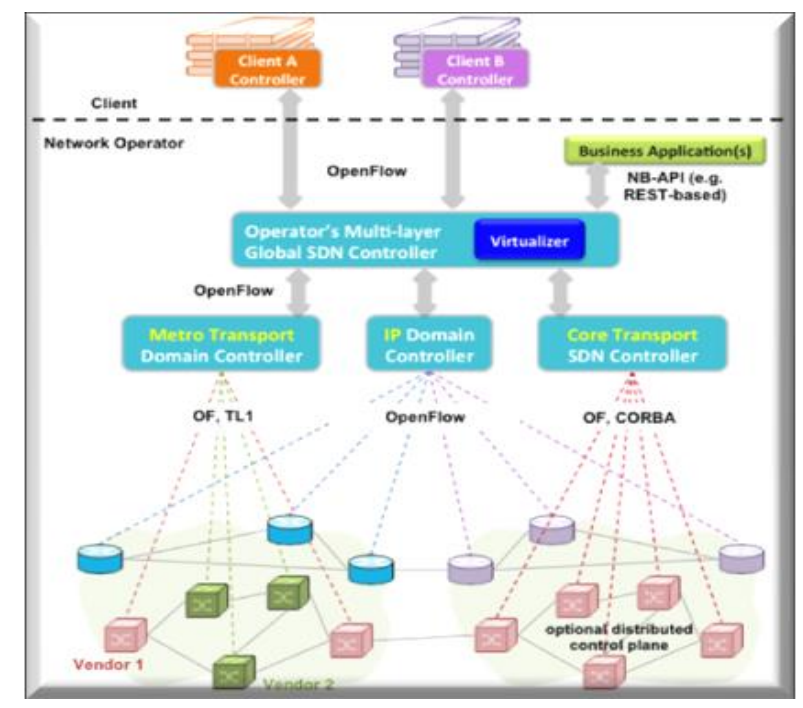

Fig. 1. Hierarchical control architecture.

In this architecture, OpenFlow is not just extended from packet domain to TDM circuit, it can support Flexible Grid DWDM, include optical power equalization, impairments etc. 


\section{INNOVATIVE RESEARCHES FOR INTELLIGENT OPTICAL TRANSPORT NETWORK}

In recent years, many innovative researches and experimental testbeds were on the way to meet ultimate mature and standardized optical SDN network.

In [2], it suggested an OF extension using a generic OF agent to be placed on the optical node to enable it to support OF protocol. In [3], it compared the distributed and centralized control methods and suggests an SDN application for multilayer network. In [4], it introduced a novel unified control plane for multi-domain and multi-transport networks based on SDN framework with pure OpenFlow as a protocol enabler, and also compared it with hybrid GMPLS and OpenFlow approach which taking advantages of the rich network control functionalities of GMPLS, then evaluate the blocking rate, path setup time, hardware setup time and throughput performance for the two approaches, the results shown pure OpenFlow got better performance. Other SDN application such as access networks and data center were studies on [5] and [6] respectively. In [7], the author extended OpenFlow protocol to be that can recognize the spectrum fragments situation and can carry defragmentation results from the controller, and the experimental testbed verified such optical SDN architecture could improve the resource utilization and decrease of block rate. In [8], it proposed extension to OpenFlow protocol to realize QOS-aware control plane for optical burst switch (OBS), and the simulation evaluation comparing with conventional GMPLS distributed protocol demonstrated that the proposed OpenFlow protocol optimizes the network performance in terms of network throughput, burst blocking probability, and packet latency while offering better Quality of Service to various classes of traffic.

From the above experimental testbeds results, what should be addressed here again is, although GMPLS still play important roles on current optical network management and give much feasibility for experimental tests of optical SDN/OpenFlow networks, we think it's an interim part of optical SDN networks. Today, GMPLS can be found in some core networks and sometimes in the transport network, but it is essentially not available in the access network. In addition, GMPLS is not typically interoperable across vendors. SDN is a born good choice network structure for multi-domain, multi-layer and multi-vendor networks.

As for actual detailed major jobs to build an intelligent SDN/ OpenFlow based optical transport networks, it include SDN virtual transport platform, many detailed supports include various software modules, algorithms and mechanisms for abstraction, virtualization and mapping.

\section{A. SDN Virtual Transport Platform}

An SDN virtual transport platform plays the role of resource abstraction to virtualize transmission pipes, enabling the communication network to function like an IT system with programming and versatile control capabilities. This platform should let users can manage and control their own virtual networks, or create new and innovative services using the following applications:

- The Virtual Transport Service application dynamically creates multiple virtual networks on demand to meet individual customer needs, all from the same physical network. Each customer can route circuits and provision services over their own virtual network. The application also allows a customer to add or remove capacity as needed, without conflicting with other tenants or the physical network.

- The Flexible Optical Network Control application can enable automatic on-demand adjustment in modulation format and line rate based on user's need, resulting in optimally utilized network resources with zero wasted bandwidth.

- The Network Health Analysis application can track network resource utilization, performance KPIs and alarms in real time, and provide survivability analysis on demand. It functions as a service degradation warning mechanism.

Our survey result in that On-Demand Secure Circuits and Advance Reservation System (OSCAR), provided by ESnet, may be a good choice for creating this platform because it is playing a vital role in the development of OpenFlow, a relatively new SDN protocol that allows the path of network packets through the network of switches to be determined by software running on the network routers.

\section{B. Innovation Algorithms and Mechanisms}

The above described platform needs more detailed algorithms and mechanisms for abstraction and virtualization to support and fulfill a unified intelligent control plane. Here we survey some innovative jobs as following.

In [4], it also developed an algorithm bundle, including several algorithms designed for different network slicing scenarios (i.e. slicing over single/multiple fixed-grid network domains, single/multiple flexi-grid network domains, mixed fixed- \& flexi-grid network domains), which is running on top of the OF controller to compose virtual network (VN) slices over flexi and fixed-grid domains. The algorithm supports two main functionalities, one is to calculate the best path from source to destination for all connectivity within each VN slice and the latter is to find the optimum spectrum across domains to fulfil bandwidth requirements of each $\mathrm{VN}$ slice request.

In [7], it also detailed a defragmentation algorithm, this algorithm rank the existing connections according to their indexes of occupied frequency slots in an increasing order $C=$ $\left\{c_{i}\right\}$, then record the occupied frequency slot index $f_{i}$ spectrum bandwidth $b_{i}$, and hop count $h_{i}$ of $c_{i}$ in $C$, then create virtual network topology, and calculate its hop number $h_{i}{ }^{\prime}$, if the $h_{i}{ }^{\prime}$ become the minimum one, then can assign the $b_{i}$ to $c_{i}$ and remove $c_{i}$ from $C$. And in [9], the similar method is used as optical grooming algorithm with Spectrum Engineering view.

In [8], the QoS-aware unified control protocol also consists of adaptive burst assembling algorithm, latency-aware burst routing and scheduling algorithm. In the proposed adaptive-burst assembling algorithm, bursts are assembled at edge nodes while the burst size is estimated at the controller, the controller estimates the burst size based on the available network resources and the latency requirement of a traffic class; the latency-aware burst routing and scheduling algorithm considers the network state information and application latency requirements in determining the burst routing and scheduling while observing the wavelength 
continuity constraint.

In [10], it presents an efficient algorithm for mapping a virtual optical network topology onto a physical multi-domain optical network with the objective of minimizing the total network link cost. It divided the problem into two parts. The first part involves selecting an appropriate TA scheme. In our problem, the aggregated topology should not only provide connectivity between border nodes, but should also show connectivity to the physical nodes that serve as candidate nodes for the VON, furthermore, intra-domain link cost information should be reflected in the aggregated links. The second part of the problem involves determining the mapping of virtual nodes to candidate physical nodes in the various domains, and determining the physical routes for each virtual link.

In [11], it proposed an auxiliary-graph based algorithm to map virtual infrastructures with survivability over T-SDN, which adopts modified Suurballe's algorithm to find a parallel mapping of the primary and backup VIs over the physical substrate. As comparing with sequential VI mapping algorithm, the parallel VI mapping algorithm significantly enhances the blocking performance of VI demands, and thus improves the traffic-carrying capacity of networks.

And more efforts are continuing to focus on software modules, algorithms and mechanisms of optical transport SDN to build a solid and unified intelligent optical transport SDN.

\section{FUTURE TECHNOLOGIES TO IMPROVE INTELLIGENCE FOR OPTICAL SDN}

As the Open Network Foundation still not finalize the architecture and OF extension for L0-L1 circuit switch and abstraction of L2 for optical transport network, we think QOS, protection and restoration etc. will be focused on the optical layers and control plane of optical transport network and it will involve more intelligence technologies. And silicon photonic technology will let optic layer include transceiver and routing more cost-efficient, programmable and intelligent-controlled.

\section{CONCLUSION}

In this paper, we use the intelligence views to illustrate SDN accompanied with OpenFlow protocol as a good choice to solve the challenges for optical transport network in new telecom applications. And detailed how the new optical technologies and intelligent SDN unified control plane can solve such challenges.

Our major contributions in this paper are that we surveyed many innovative researches for building SDN/OpenFlow optical transportation network architectures, creating the control plane platform and developing different detailed algorithms and mechanisms to support and optimize the SDN optical transportation networks; and summarized these researches and gave some suggestions, we think it is valuable for optical transport SDN network researchers.

\section{REFERENCES}

[1] M. Auster, S. Balakrishnan, and M. McBride, (May, 2014) OpenFlow-enabled Transport SDN, Open Networking Foundation. [Online] pp. 1-16. Available: https://www.opennetworking.org/images/stories/downloads/sdn-resou rces/solution-briefs/sb-of-enabled-transport-sdn.pdf

[2] D. Simeonidou, R. Nejabati, and M. P. Channegowda, "Software defined optical networks technology and infrastructure: enabling software-defined optical network operations," IEEE/OSA Journal of Optical Communications and Networking, vol. 5, pp. A274-A282, Oct. 2013.

[3] S. Gringeri, N. Bitar, and T. J. Xia, "Extending software defined network principles to include optical transport," IEEE Communications Magazine, vol. 51, pp. 32-40, Mar. 2013.

[4] M. Channegowda, R. Nejabati, M. R. Fard, and S. Peng, "Experimental demonstration of an OpenFlow based software-defined optical network employing packet, fixed and flexible DWDM grid technologies on an international multidomain testbed," Optic Express, vol. 21, pp. 5487-5498, Mar. 2013.

[5] N. Cvijetic, A. Tanaka, P. N. Ji, K. Sethuraman, and S. Murakami, "SDN and openflow for dynamic flex-grid optical access and aggregation networks," Journal of Lightwave Technology, vol. 32, pp. 864-870, Feb. 2014

[6] Y. L. Zhao, J. Zhang, H. Yang, and X. S. Yu, "Data center optical networks (dcon) with openflow based software defined networking (SDN)," in Proc. 2013 8th International ICST Conference on Communications and Networking in China (CHINACOM), Aug. 2013, pp. 771-775.

[7] X. S. Yu, Y. L. Zhao, J. Zhang, and L. N. Gao, "Spectrum defragmentation implementation based on software defined networking (sdn) in flexi-grid optical networks," in Proc. 2014 International Conference on Computing, Networking and Communications, Optical and Grid Networking Symposium, Feb. 2014, pp. $502-505$

[8] A. N. Patel, P. N. Ji, and T. Wang, "QoS-aware optical burst switching in openflow based software-defined optical networks," in Proc. 2013 17th International Conference on Optical Network Design and Modeling (ONDM), April 2013, pp. 275-280.

[9] X. S. Yu, Y. L. Zhao, and J. Zhang, "Optical grooming with spectrum engineering (og-se) in flexi-grid networks," presented at the Optical Fiber Communication Conference, San Francisco, California, March 9-13, 2014.

[10] S. J. Hong, J. P. Jue, and Q. Zhang, "Effective virtual optical network embedding based on topology aggregation in multi-domain optical networks," presented at the Optical Fiber Communication Conference, San Francisco, California, March 9-13, 2014.

[11] Z. Ye, A. N. Patel, and P. N. Ji, "Survivable virtual infrastructure mapping over transport software-defined networks (T-SDN)," presented at the Optical Fiber Communication Conference, San Francisco, California, March 9-13, 2014.

Xu Chen received the M.S. degree from University of Electronic Science and Technology of China (UESTC) in 2003 with the major of optical communication.

$\mathrm{He}$ had worked in many telecom companies delivering optical transceivers, optical network equipment and telecom services. Now he leads a team in the Global Technology Center for Premier Farnell in Chengdu, China. His research interests include new technologies in optical communication about silicon photonic, NG-PON, SDN etc.

Yongjian Zhang received the B.S. and M.S. degrees in electrical engineering from University of Electronic Science and Technology of China (UESTC) in 1997 and 2003, respectively.

He was a member of research staff at China Institute of Communications. In 2003, he joined the Faculty of Information and Science Technology, University of International Relations, where he is currently an associate professor. His research interests include communication theory, signal processing, and wireless and mobile communication. 